\title{
Strangelets, strangeness and antimatter: results from the experiment NA52 at CERN
}

\author{
S. Kabana* and G. Ambrosini, R. Arsenescu, H.P. Beck, K. Borer, P. Hess, \\ R. Klingenberg, G. Lehmann, R. Mommsen, U. Moser, K. Pretzl, J. Schacher, \\ R. Spiwoks, F. Stoffel, M. Weber ${ }^{\dagger}$ \\ University of Bern, Sidlerstrasse 5, 3012 Bern, Switzerland \\ E-mail: 'sonja.kabana@ecern.ch' \\ C. Baglin,A. Bussière,J.P. Guillaud \\ CNRS-IN2P3, LAPP Annecy, F-74941 Annecy-le-Vieux, France
}

\section{K. Elsener,K.D. Lohmann}

CERN, SL Division, CH-1211 Geneva 23, Switzerland

\section{Ph. Gorodetzky}

PCC - College de France, 11 Place Marcellin Berthelod, 75005 Paris, France

\section{T. Lindén,J. Tuominiemi}

Dept. of Physics, University of Helsinki, PO Box 9, FIN-00014 Helsinki, Finland

\begin{abstract}
We present results of the CERN experiment NA52 which measures $\mathrm{Pb}+\mathrm{Pb}$ collisions at $158 \mathrm{~A} \mathrm{GeV}$. The topics we address are: 1. strangelet search, 2. nuclei and antinuclei production and 3. strangeness production. No strangelets have been found. We set upper limits which stretch down to the minimal values of $\sim 210^{-10}$ for negatively and $310^{-9}$ for positively charged strangelets. We observed $5 \overline{{ }^{3} \mathrm{He}}$ near midrapidity. Nuclei and antinuclei cross sections suggest production mainly through coalescence, out of a thermalised particle source, at the time of the thermal freeze-out as witnessed by their temperature of 100-120 MeV. Charged kaons per participant nucleon $\left(N_{p}\right)$ show an onset of enhancement followed by saturation at $N_{p} \sim 80$, corresponding approximately to an initial energy density of $\epsilon_{i} \sim 1.3 \mathrm{GeV} / \mathrm{fm}^{3}$. This onset may be connected to the onset of the QCD phase transition.
\end{abstract}

KEYworDs: Quark gluon plasma, strangelets, strangeness, antimatten.

${ }^{*}$ Speaker.

${ }^{\dagger}$ We thank the Schweizerischer Nationalfonds for their support. 


\section{Introduction}

NA52 is a fixed target experiment at the CERN SPS with the main goal to search for strangelets in $158 \mathrm{~A} \mathrm{GeV} / \mathrm{c} \mathrm{Pb}-\mathrm{Pb}$ collisions. For a description of the experiment and of

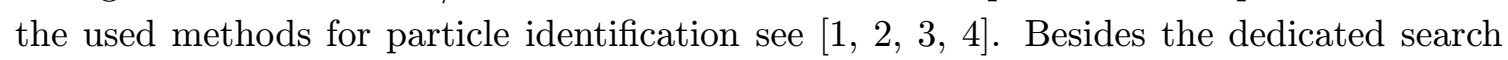

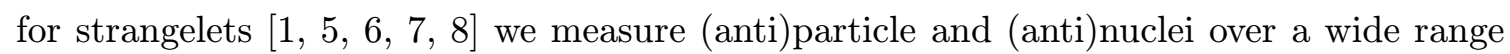

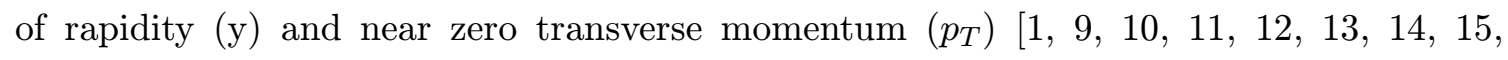
12. and (anti)particle and (anti)nuclei production cross sections in $\mathrm{Pb}+\mathrm{Pb}$ collisions at 158 A GeV. Strangelets are exotic hadrons made up by more than 3 quarks, stabilized by a high s-quark content. Particle and antiparticle production, and in particular antimatter and strange particle production, are important for searches for the onset of the QCD phase

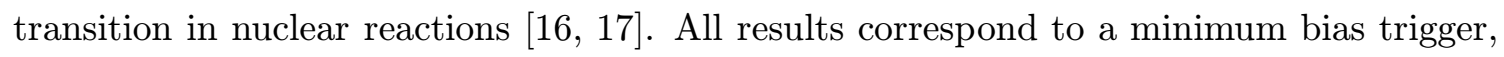
unless differently stated.

\section{Strangelet search}

No charged strangelets have been found in the full set of data. The results we present here, include the data from the last NA52 run in 1998, and are the final NA52 results [i] 1 ind. Figure $]_{1}^{1}$ shows the upper limits for negatively charged strangelets that we set. For the positively charged see reference [i]1].

\section{Particle and Antiparticle cross sections}

Figure $\overline{2}_{1}^{\overline{1}}$ shows the production cross sections for negatively charged particles as a function of rapidity [i] 1 in]. The filled colored dots are from data taken in 1998. We observed in total $5 \overline{{ }^{3} \mathrm{He}}$ antinuclei. As shown in figure 2 of $[9 \overline{9}]$ the cross sections of nuclei and antinuclei at

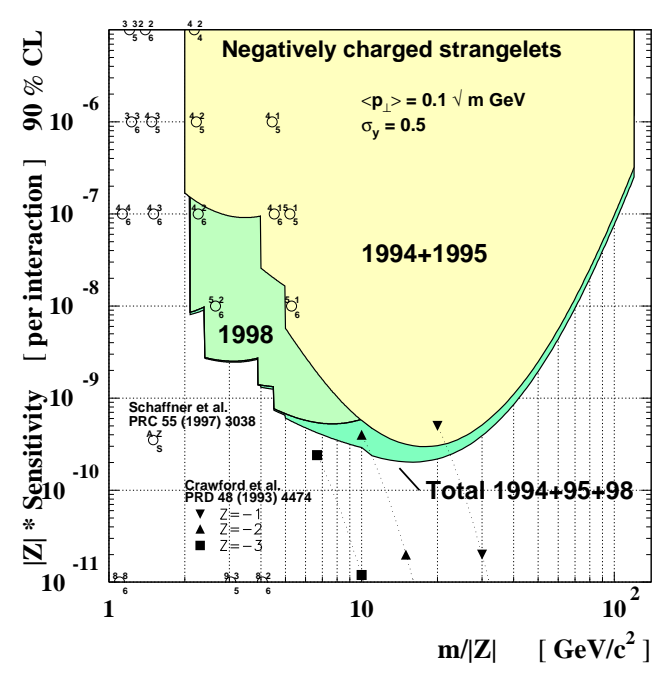

Figure 1: Upper limits set for the production of negatively charged strangelets in $\mathrm{Pb}+\mathrm{Pb}$ collisions at $158 \mathrm{~A} \mathrm{GeV}$.

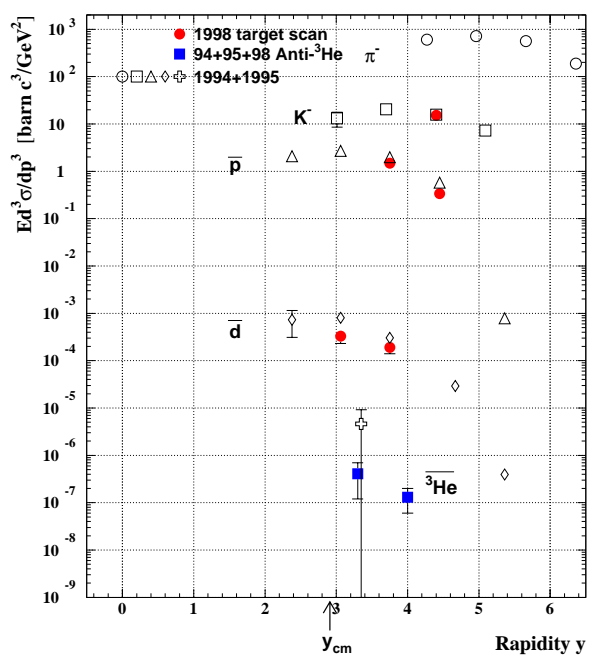

Figure 2: Invariant cross section of antiparticles and antinuclei in $\mathrm{Pb}+\mathrm{Pb}$ collisions at $158 \mathrm{~A} \mathrm{GeV}$ at $p_{T} \sim 0$, as a function of rapidity. 
rapidity $\mathrm{y}=3.3$ follow the predictions of a simple coalescence model. Additionally a function of the form constant $_{1} /$ constant $_{2}^{A-1}$, fits the dependence of particle cross sections on A (see EPS2001 talk) respectively on $\mathrm{m}$ (see figures 6 and 7 in [i-i]) at rapidities $\mathrm{y}=3.3$ and 5.4. The constant $_{2}$ can be interpreted as 'coalescence penalty factor' [i1 $\left.\overline{1} \overline{1}\right]$. However as can be seen in the above pictures we find constant $_{2}$ factors which are different for positively and negatively charged particles at $y=3.3$. To understand the origin of this difference, we compare the same data with the predictions of a thermal model. Figures $\underline{3}_{1}, \underline{4}_{1}$ and demonstrate that the invariant cross sections as a function of $m_{T}$ are well described by a thermal model fit. The fit function is an approximation of the Boltzman equation, where we substitute A by (mass/GeV): $E \frac{d^{3} \sigma}{d p^{3}} / E(2 S+1)=c e^{-m_{T}\left(\cosh y+\left(\frac{\mu_{B}}{T}\right) T / G e V\right) / T}$. We fix the ratio $\mu_{B}$ (proton) $/ T$ to its value extracted from the $\bar{p} / p$ ratio, namely 1.4 for $\mathrm{y}=3.3$ and 6.3 for $\mathrm{y}=5.4$. We use in the fit $+\left|\mu_{B} / T\right|$ for the positive and $-\left|\mu_{B} / T\right|$ for the negative particles. Notably, the temperature which can be obtained from the yields of both positively and negatively charged particles at $\mathrm{y}=3.3$ comes out to be $\mathrm{T} \sim 120 \mathrm{MeV}$. Therefore, the difference in the slopes of positively and negatively charged particles at the same rapidity $\mathrm{y}=3.3$, as seen in the figures $\overline{3}$, and $\overline{4}$, can be explained as due to the different sign of the baryochemical potential for positively and negatively charged particles. Note that these temperatures are affected by the presence of $p_{T}$ dependent dynamic phenomena like the transverse flow. The thermal and the coalescence interpretations are both valid. After the thermal freeze-out of $p, \bar{p}, n$ and $\bar{n}$ at a $\mathrm{T}$ of $\sim 170 \mathrm{MeV}$, they can coalesce to form nuclei and antinuclei. However newly formed nuclei and antinuclei are weekly bound and can break up due to interaction and annihilation processes in the hadron dense environment before they finally freeze out at a $\mathrm{T}$ of $\sim 120 \mathrm{MeV}$. The resulting $\mathrm{T}$ of $\sim 120 \mathrm{MeV}$ at $\mathrm{y}=3.3$

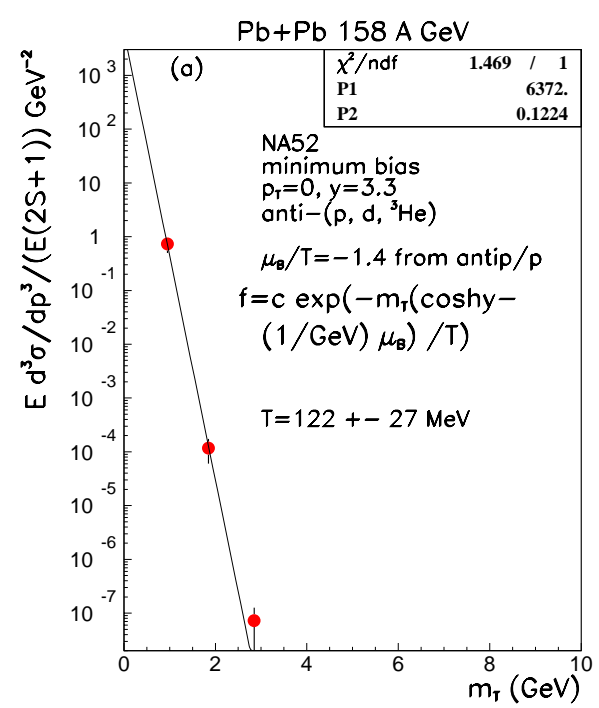

Figure 3: Invariant cross section as a function of $m_{T}\left(m_{T}=m\right.$ for $\left.p_{T}=0\right)$ for negatively charged particles at $\mathrm{y}=3.3$ and $p_{T} \sim 0$ in $\mathrm{Pb}+\mathrm{Pb}$ collisions at $158 \mathrm{~A} \mathrm{GeV}$.

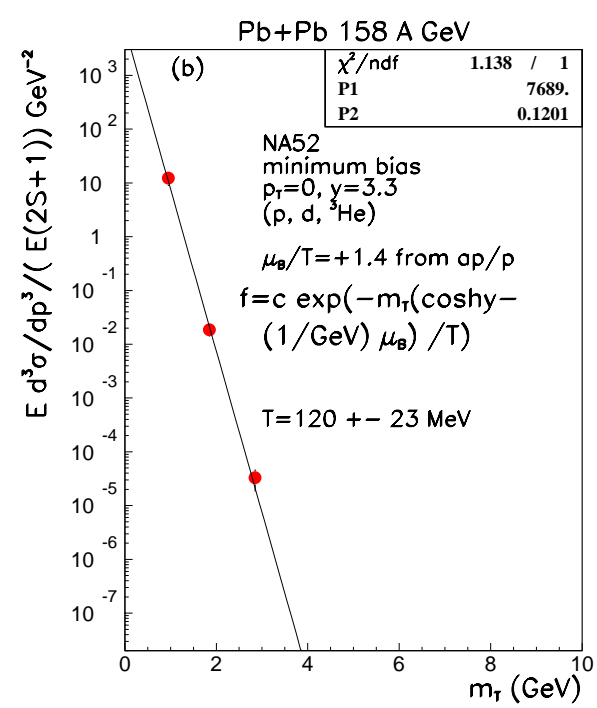

Figure 4: Invariant cross section as a function of $m_{T}\left(m_{T}=m\right.$ for $\left.p_{T}=0\right)$ for positively charged particles at $\mathrm{y}=3.3$ and $p_{T} \sim 0$ in $\mathrm{Pb}+\mathrm{Pb}$ collisions at $158 \mathrm{~A} \mathrm{GeV}$. 


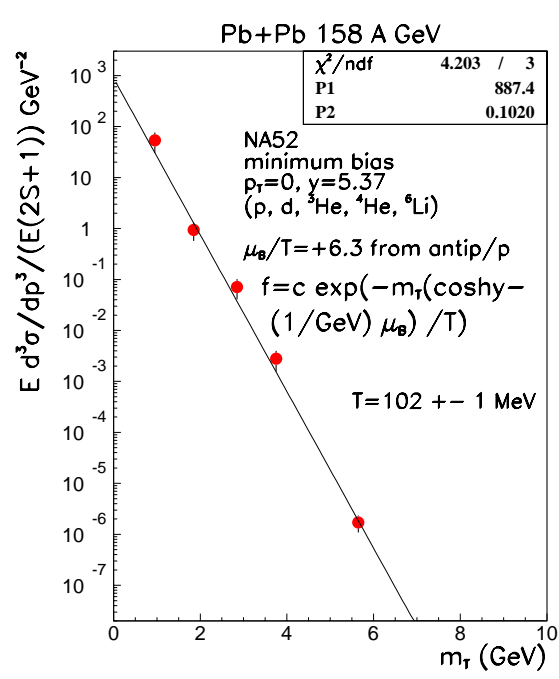

Figure 5: Invariant cross section as a function of $m_{T}$ for positively charged particles at $\mathrm{y}=5.4$ and $p_{T} \sim 0$ in $\mathrm{Pb}+\mathrm{Pb}$ collisions at 158 A GeV.

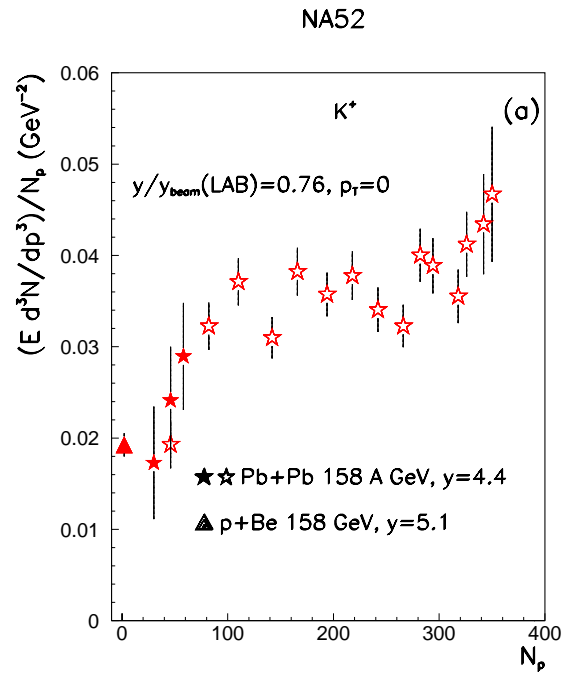

Figure 6: Invariant $K^{+}$yields at $\mathrm{y}=4.4$ and $p_{T} \sim 0$ in $\mathrm{Pb}+\mathrm{Pb}$ collisions at $158 \mathrm{~A} \mathrm{GeV}$, normalized to the number of participant nucleons $\left(N_{p}\right)$, as a function of the latter.

(see fig. 3 and 4) respectively of $\sim 100 \mathrm{MeV}$ at $\mathrm{y}=5.4$ (see fig. $\underline{\underline{p}}_{1}^{\prime}$ ) are of the same order, which supports the conclusion that nuclei and antinuclei are formed through coalescence just before they thermally freeze out.

\section{Strangeness}

Figure ' $6 \overline{6}$ ' demonstrates that charged kaon yields show a threshold behaviour at $N_{p} \sim 80$

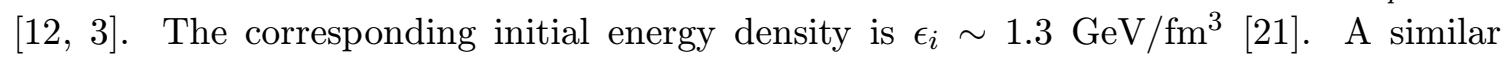
behaviour has been seen in $\Xi$ and $\bar{\Xi}$ by NA57 [20 $\overline{0}^{\eta}$. This threshold is visible not only in $\mathrm{Pb}+\mathrm{Pb}$ collisions at $158 \mathrm{~A} \mathrm{GeV}$ but also in other $\mathrm{A}+\mathrm{A}$ colliding systems at different energies [2 $2 \overline{1}]$. This is also observed in the temperature and the strangeness suppression factor $\lambda_{s}$ when investigated as a function of $\epsilon_{i}$ under conditions of a common chemical potential [2를. These phenomena may be interpreted as due to the onset of the QCD phase transition near $\epsilon_{i} \sim 1.3 \mathrm{GeV} / \mathrm{fm}^{3}$.

\section{Conclusions}

The NA52 experiment at the CERN SPS searched and found no evidence for multiquark bound states called strangelets in $\mathrm{Pb}+\mathrm{Pb}$ collisions at $158 \mathrm{~A} \mathrm{GeV}$. Upper limits were set, reaching down to a sensitivity of $210^{-10}$ for negatively charged particles. We found evidence that nuclei and antinuclei are produced mainly through coalescence temperature extracted from the $m_{T}$ dependence of nuclei and antinuclei at midrapidity $(\mathrm{y}=3.3)$ is $\sim 120 \mathrm{MeV}$, while at forward rapidity $(\mathrm{y}=5.4) \mathrm{T} \sim 100 \mathrm{MeV}$. The data are in good agreement with both coalescence model and thermal model predictions. The opposite sign of the chemical potential explains the different slopes of positively and negatively charged particles at $y=3.3$. These results are shown here for the first time. We found 
evidence that the onset of strangeness enhancement takes place in $\mathrm{Pb}+\mathrm{Pb}$ collisions at $N_{p}$ $\sim 80$, respectively at an initial energy density of $1.3 \mathrm{GeV} / \mathrm{fm}^{3}$. The same behaviour can be found not only in one system $(\mathrm{Pb}+\mathrm{Pb})$ as a function of centrality, but also in other $\mathrm{A}+\mathrm{A}$ data at different energies $\left[\overline{2} \overline{1} \overline{1}_{i}^{1}, \overline{2} \overline{2} \overline{2}\right]$. This onset may be interpreted as due to the onset of the QCD phase transition, while more data from SPS and RHIC are needed to confirm this interpretation.

\section{References}

[1] M. Weber et al., (NA52), J. of Phys. G 27, 3, (2001), 487.

[2] M. Weber et al., (NA52), Nucl. Phys. A 661 (1999) 177c.

[3] G. Ambrosini et al., (NA52 coll.): J. of Phys. G 27, 3, (2001), 495 and hep-ph/0010053, and paper submitted to ICHEP2000 and hep-ph/0010045.

[4] K. Pretzl et al., (NA52), World Scientific (1995) 230.

[5] G. Appelquist et al., (NA52), Phys. Rev. Lett. 76 (1996) 3907.

[6] F. Stoffel et al., (NA52), Heavy Ion Physics 4 (1996) 429.

[7] R. Klingenberg et al., (NA52), Nucl. Phys. A 610 (1996) 306c.

[8] R. Klingenberg et al., (NA52), Proc. of Renc. de Moriond, QCD 22-29 March 1997, Les Arc 1800, France, 497.

[9] K. Pretzl et al., (NA52), proceedings of the 'Symposium on Fundamental Issues in elementary Matter', 25-29 September 2000, Bad Honnef, Germany, published in Acta Physica Hungarica, nucl-ex/0011016.

[10] S. Kabana et al., (NA52), Nucl. Phys. A 638 (1998) 411c, S. Kabana et al., (NA52), J. of Phys. G 23 (1997) 2135.

[11] M. Weber et al., (NA52), Proceedings of the VII International Conference on Calorimetry in High Energy Physics, 9-14 Nov. 1997, Tucson, Arizona, USA, S. Kabana et al., (NA52), J. Phys. G 25 (1999) 217, G. Ambrosini et al., (NA52), New J. of Phys. 1, 23, 1999.

[12] G. Ambrosini et al., (NA52), New J. of Phys. 1, 22, 1999. S. Kabana et al., (NA52), Nucl. Phys. A 661 (1999) 370c.

[13] R. Arsenescu et al., (NA52), J. Phys. G 25 (1999) 225.

[14] G. Ambrosini et al., (NA52), Phys. Lett. B 417 (1998) 202. 1994.

[15] G. Appelquist et al., (NA52), Phys. Lett. B 376 (1996) 245.

[16] J. Ellis et al., Phys. lett. B 233 (1989) 223,

[17] P. Koch et al, Phys. Rep. 142, (1986), 167.

[18] M. Weber, PhD Thesis, University of Bern, 2001, Switzerland.

[19] T.A. Armstrong et al. (E864), Phys. Rev. Lett. C61: 064908 (2000), nucl-ex/0003009.

[20] N. Carrer et al., (NA57), Proceedings of the QM2001 conference, Stony Brook, January 2001.

[21] S. Kabana, New J. of Phys. 3 (2001) 16 and hep-ph/004138, S. Kabana, J. of Phys. G 27, 3, (2001), 497 and hep-ph/0010228, S. Kabana, Proc. ICHEP2000 and hep-ph/0010246.

[22] S. Kabana, P. Minkowski, New J of Phys 3 (2001) 4 and hep-ph/0010247, S. Kabana, Eur. Phys. J. C21, 3, (2001), 545 and hep-ph/0104001, S. Kabana, proc. Renc. de Moriond QCD 2001 and hep-ph/0105152. S. Kabana, proc. of the ISMD2001 conference, China, Sept. 2001. 